\title{
El clúster como modelo factible para el desarrollo del sector de confecciones-diseño de moda: un estudio en Bucaramanga (Colombia)
}

The Cluster as a Feasible Model for the Development of the Tailoring-Fashion Design Industry: A Study in Bucaramanga (Colombia) O cluster como modelo factível para o desenvolvimento do setor de confecçõesdesenho de moda: um estudo em Bucaramanga (Colômbia)

\section{Laura Barajas ${ }^{*}$ \\ Diana Oliveros ${ }^{* *}$}

Fecha de recibido: 25 de julio de 2013

Fecha de aprobado: 22 de abril de 2014

Doi: dx.doi.org/10.12804/rev.univ.empresa.27.2014.09

Para citar este artículo: Barajas, L., \& Oliveros, D. (2014). El clúster como modelo factible para el desarrollo del sector de confecciones-diseño de moda: un estudio en Bucaramanga (Colombia). Universidad \& Empresa, 16(27), 259-280. doi: dx.doi.org/10.12804/rev.univ.empresa.27.2014.09

* Profesional en Administración de Empresas de la Universidad Autónoma de Bucaramanga (Bucaramanga, Colombia). Asian Market Representative en EXICOB S.A.S. (Bucaramanga, Colombia - Chengdu, China). Correo electrónico: lbarajas2@unab.edu.co

** Doctora en Sistemas Flexibles de Dirección de Empresas del Departamento de Gestión de Empresas de la Universidad Pública de Navarra (España). Docente Titular del programa de Administración de Empresas de la Universidad Autónoma de Bucaramanga (Colombia). Correo electrónico: doliveros364@unab.edu.co 


\section{RESUMEN}

En los últimos años se ha extendido la aplicación del modelo de clústeres en diferentes ámbitos empresariales como una alternativa para mejorar la competitividad. Este estudio tiene como objetivo determinar la factibilidad de la aplicación del clúster en el sector de confecciones-diseño de moda de Bucaramanga (Colombia). Este sector, si bien es uno de los más tradicionales de la región, actualmente se encuentra rezagado. En la investigación se realizan encuestas y entrevistas en las micro, pequeñas y medianas empresas (Mipymes) del sector en esta ciudad. Este material es analizado por medio de 1) la metodología del diamante de Porter y 2) las cinco fuerzas competitivas (planteadas por el mismo autor). Los resultados muestran que el clúster es un modelo factible para mejorar la competitividad de este sector en Bucaramanga.

Palabras clave: cadena productiva, clúster, competitividad, confecciones.

\section{ABSTRACT}

In recent years, application of the clusters model in different entrepreneurial environments has been increasingly used as an alternative to improve competitiveness. The aim of this study is to determine the feasibility of the application of the cluster in tailoring-fashion design industry in Bucaramanga (Colombia). This industry, while it is one of the most traditional of the region, has now fallen behind. Surveys and interviews in small and mediumsized enterprises (SMEs) of the industry in this city were held during the research. This material is analyzed through 1) Porter's diamond methodology and 2) the five competitive forces (put forward by the same author). The results shows that the cluster is a feasible model with which to make this sector in Bucaramanga more competitive.

Keywords: Competitiveness, cluster, supply chain, tailoring.

\section{RESUMO}

Nos últimos anos se tem estendido a aplicação do modelo de clusters em diferentes Âmbitos empresariais como uma alternativa para melhorar a competitividade. Este estudo tem como objetivo determinar a factibilidade da aplicação do cluster no setor de confecçõesdesenho de moda de Bucaramanga (Colômbia). Este setor, se bem é um dos mais tradicionais da região, atualmente encontra-se atrasado. Na pesquisa realizam-se enquetes e entrevistas nas micro, pequenas e médias empresas (mipymes) do setor nesta cidade. Este material é analisado através de 1) a metodologia do diamante de Porter e 2) as cinco forças competitivas (expostas pelo mesmo autor). Os resultados mostram que o cluster é um modelo factível para melhorar a competitividade deste setor em Bucaramanga.

Palavras-chave: cadeia produtiva, cluster, competitividade, confecções. 


\section{INTRODUCCIÓN}

Si bien la globalización y las políticas de libre intercambio han tenido incidencias negativas en las industrias menos preparadas en los últimos años, también han sido precursoras de cambios y transformaciones en las empresas alrededor del mundo. El dilema planteado por este fenómeno es "evolucionar o desaparecer".

Una de las herramientas que las empresas han adoptado para reaccionar ante las amenazas de la globalización es el clúster. Este modelo, por su amplio campo de aplicación y la gran variedad de literatura económica existente al respecto, ha generado alguna controversia en lo que a su definición se refiere. Para efectos de este estudio, se parte del concepto propuesto por Porter (2008a) por el cual "un clúster es un grupo geográficamente próximo de compañías interconectadas e instituciones asociadas en un campo particular, vinculadas por elementos en común y por complementariedades" (p. 215).

El clúster ha sido aplicado en una gran diversidad de casos, en contextos diferentes, y se ha discutido acerca de las connotaciones que este puede adquirir (Navarro, 2003). Sin embargo, no es objeto de esta investigación debatir sobre el concepto en sí mismo, entre otras razones, porque hay tantas definiciones de clúster como tipos de organizaciones que usan el término en la actualidad. Por lo anterior, en este trabajo nos limitamos a reconocer que el modelo de clúster tiene implícitas múltiples dimensiones que se deben considerar a la hora de aplicarlo en la realidad. Estas son: "i) límites espaciales del cluster [sic] (nacional, regional o local); ii) tipo de relación (interdependencia o similitud) entre empresas o sectores; iii) tipo de flujos (de productos o de conocimientos); iv) organizaciones e instituciones tomados en consideración" (Navarro, 2003, p. 17).

Esta investigación pretende determinar la factibilidad de replicar dicho modelo con éxito en la ciudad de Bucaramanga y su área metropolitana, guardando las proporciones, por supuesto, y teniendo en cuenta las características particulares de su parque empresarial, su cultura organizacional y las instituciones de apoyo que existen actualmente.

\section{APLICACIONES DEL MODELO CLÚSTER EN EL MUNDO}

Canadá, una nación desarrollada, como Estados Unidos o Reino Unido, presentó el mismo comportamiento de estos países cuando las políticas de liberalización económica tuvieron auge y se convirtieron 
en una realidad. Algunas industrias que no se encontraban en una posición competitiva muy favorable se vieron en la necesidad de dinamizarse. Un estudio aplicado a empresarios del sector de confecciones de Canadá reveló que cerca del $83 \%$ de las empresas de ese país, pertenecientes a esta industria, parecían no tener una estrategia clara para dar respuesta a los cambios del mercado (e.g., el libre ingreso de productos manufacturados de confecciones provenientes de países en vía de desarrollo).

De este modo, las pequeñas y medianas empresas se vieron en la obligación de volverse más competitivas y productivas, un reto bastante difícil de asumir después de estar en un ambiente proteccionista. Las compañías comenzaron a cambiar su propuesta de valor, basada para ese entonces en la elaboración de productos manufacturados, y le dieron tanto énfasis a la calidad, la sofisticación y la innovación del producto, que se volvieron expertas en estos temas. Esto mejoró en gran medida la oferta de valor que los clientes percibían - la cual incluía el diseño del producto, el uso de nuevas tecnologías y una mayor inversión en marca-, lo que se tradujo después en un aumento de la demanda de dichos productos (Campaniaris, Hayes, Jeffrey, \& Murray, 2011).
De este modo, las empresas comenzaron a desarrollar una especie de joint venture, integrando algunos proveedores y empresas que ofrecen productos similares para ampliar su mercado (Campaniaris et al., 2011). Además, tomaron en cuenta otras instituciones como universidades (que aportan la cuota de investigación y desarrollo y generación de conocimiento) y la Toronto Fashion Incubator (TFI). Hoy, las empresas canadienses de confecciones y moda basan su éxito y su ventaja competitiva ya no en la producción de las prendas, sino en la innovación, la inversión en marca y los servicios adicionales.

Se encuentra también que en Jiangsu, una provincia costera de China, se comprendió igualmente que el modelo de clúster podría ser exitoso en la medida en que lograra mejorar la competitividad del sector como un todo. Esto integrando desde el hilado de tejidos hasta la producción de prendas de vestir, a través de una afiliación voluntaria, pero conservando la independencia jurídica y la autonomía gerencial de cada empresa asociada (Mercado, Fontalvo, \& De la Hoz Granadillo, 2011).

China, por su parte, tiene unos niveles de producción basados en grandes lotes manufactureros que permiten un mayor aprovechamiento de los re- 
cursos y la aplicación de economías de escala. Estos son factores determinantes a la hora de plantear sus estrategias competitivas. Además, el papel que juega el Gobierno es de suma importancia, ya que este incentiva la inversión extranjera y otorga subsidios para activar el desarrollo de distintas provincias para conseguir que estas sean de categoría mundial. Gracias a esto, el sector textil-confecciones produce cerca de $35 \%$ de los ingresos de la provincia y el $22 \%$ del total de las exportaciones (Mercado et al., 2011).

EnLatinoamérica existe, entre otros, el clúster de confecciones de $\mathrm{Za}$ potlanejo (México), que cuenta con 720 empresas y que abarca 33 manzanas del distrito. Este se caracteriza por un alto grado de especialización y un proceso productivo fraccionado, en donde las empresas dueñas de la materia prima (y encargadas del diseño y la moldería) subcontratan a pequeñas fábricas maquiladoras que se dedican a la confección (siendo ese su producto terminado). Las prendas regresan después a la compañía inicial para ser etiquetadas, planchadas y comercializadas. De este modo, una vez hecha la inspección correspondiente, los productos se envían a las distintas plazas comerciales nacionales (Cota, 2009). Si bien este modelo de negocios representa ven- tajas en cuanto a costos de producción, también tiene desventajas, en particular, en relación con la calidad de los productos.

\section{APLICACIONES DEL MODELO CLÚSTER EN COLOMBIA}

El sector de confecciones-diseño de moda es uno de los más tradicionales de Colombia. Incluso, hoy, se busca posicionar a este sector como uno de clase mundial. Si bien esta cadena productiva ha presentado transformaciones y ha sido de vital importancia en el desarrollo económico del país, en la actualidad se encuentra rezagada y no ha logrado desarrollar el grado de productividad y de competitividad requerido para competir de manera adecuada.

Existen varios factores que dificultan este desarrollo, entre los que se encuentran los subsidios internacionales a la producción de algodón, el contrabando e importación de textiles y las prendas de vestir de bajo costo. Además, la firma de tratados de libre comercio es cada vez más frecuente. Esto representa una serie de amenazas, por cuanto este tipo de acuerdos constituyen barreras indirectas (no arancelarias) para múltiples sectores de países relativamente pequeños, como Colombia, que todavía no han alcanzado los estándares 
requeridos para exportar y acceder a mercados internacionales altamente competitivos.

A pesar de lo anterior, el sector que agrupa la producción textil, confecciones y diseño de moda sigue siendo relevante para la economía. En 2011, este sector representó el $17 \%$ del PIB industrial del país, siendo el área metropolitana de Bogotá el mayor productor de textiles $(50,8 \%)$ y Medellín y el Valle de Aburrá los mayores productores de confecciones (42,5\%) (Departamento Administrativo Nacional de Estadística [DANE], 2010; Inexmoda, 2012).

Un caso de éxito es Medellín, que ha sido por tradición el principal foco de desarrollo de la industria textil y de confecciones en Colombia. Allí se fue dando inicialmente una aglomeración de empresas espontánea y, hoy, es un clúster que integra toda la cadena productiva, desde la elaboración de textiles de tejido plano y de punto, a partir de las fibras de algodón y otras fibras sintéticas, pasando por la confección de las prendas, hasta la comercialización de las mismas. En la actualidad, muchas empresas se dedican a fabricar por completo prendas de vestir de reconocidas marcas extranjeras como Gap, Victoria's Secret, Nike, Abercrombie \& Fitch, Polo Ralph Lauren, entre otras. Esto obteniendo unos márgenes de ganancias superiores a los que se lograrían con el modelo de maquila, añadiendo, en el proceso, un mayor valor en todos los eslabones de la cadena productiva (Carmona \& Gil Quintero, 2010). Es necesario indicar, en este punto, que, de acuerdo con el Instituto para la Exportación y la Moda (Inexmoda, 2012), la producción nacional de textiles y prendas de vestir está destinada en un $78 \%$ al mercado interno y, el excedente, a la exportación.

En Santander, como en el resto del país, la caracterización del sector está dada, en su mayoría, por las micro, pequeñas y medianas empresas (Mipymes; en un 96\%, según el DANE citado en Compite 360, s.f.). Estas, por su naturaleza, no cuentan usualmente con una estandarización rigurosa ni con un nivel de innovación y desarrollo que las impulse y las haga sobresalir. Su estructura financiera, de hecho, no es por lo general tan fuerte como para costear tal nivel de desarrollo (comprar maquinaria de última tecnología, actualizar sus procesos, etc.). Según datos de 2011, el área metropolitana de Bucaramanga, produce solo el $1,6 \%$ del total de confecciones de Colombia y su balanza comercial se encuentra en un déficit que alcanza los USD 580000 (Encuesta Anual Manufacturera [EAM] del DANE, 
2010). Sin embargo, esta industria representa el $10,5 \%$ de las exportaciones totales del Departamento de Santander.

Un elemento adicional, que permite terminar de esbozar la situación actual del sector, consiste en aclarar que existen múltiples factores adversos que justifican su decaimiento. Entre estos se destacan tres: 1) el contrabando, 2) el desmejoramiento, en los últimos años, de las relaciones comerciales con países como Venezuela y Ecuador y 3) el ingreso de prendas de vestir y de textiles extranjeros de bajo costo. Un ejemplo, en particular, del tercero de ellos, es la llamada 'moda rápida' o fast fashion, un movimiento que está revolucionando el mundo de la moda. Este promueve un ciclo de vida más corto para los productos, con prendas de vestir que aunque cumplen con menores estándares de calidad son más baratas. La presencia de este solo hecho obliga a transformar por completo la cadena de suministro de la industria (Barnes \& LeaGreenwood, 2006).

La anterior tendencia contrasta con otra en materia empresarial conocida como la Eco-innovación. Esta se refiriere al desarrollo de múltiples innovaciones realizadas con el fin de reducir el uso de recursos naturales y la cantidad de tóxicos y sustan- cias nocivas en todas las etapas del proceso de producción; pero, además, con el objeto de hacer más amplio el ciclo de vida de los productos para reducir así su impacto ambiental (Barsoumian, Riggio, Severin, \& Van der Spek, 2011). Para lograr este tipo de innovación se hace necesaria la inversión en tecnología y el desarrollo de nuevos procesos, lo que representa elevados requerimientos de capital para las mipymes de Bucaramanga. Este es un problema que, entre otras alternativas, es posible remediar a través del modelo clúster, pues el acceso a inversión y la posibilidad de compartir información y habilidades entre empresas se dan más fácilmente cuando existen este tipo de aglomeraciones productivas. Estas, además, impulsan el desarrollo de procesos innovadores (Silva, Feitosa, \& Aguiar, 2012).

Las empresas deben prestar importancia a las macro tendencias, entre otras, a las señaladas. Estas delimitan su campo de acción y la forma en la que ellas realizan sus actividades económicas. Las condiciones de la demanda local, por ejemplo, hablan de sensibilidad a los precios por encima de las preferencias de calidad. Esto indica, entre otras opciones, que los consumidores pueden llegar a preferir los productos importados de bajo precio que las marcas colombianas. 
Un caso contrario a este fenómeno es el de Japón. Un estudio comparativo entre el comportamiento de los consumidores de este país y los de Estados Unidos muestra que los primeros parecen preferir los productos elaborados en su nación. Este hecho aumenta el consumo de prendas de vestir made in Japan y disminuye el de las importadas, considerando un aumento general, sin importar su origen, en el nivel de precios de estos productos. En cambio, en Estados Unidos, disminuye el consumo total de las prendas de vestir (tanto de las importadas como de las locales) (Lee \& Karpova, 2011).

\section{METODOLOGÍA, DATOS Y VARIABLES}

Para esta investigación se utilizaron dos recursos específicos. Por un lado, la metodología de las cin- co fuerzas competitivas de Porter, para la descripción del sector y, por el otro, el diamante de competitividad, de este mismo autor, para la comparación del posible clúster en Bucaramanga con el ya célebre ubicado en la ciudad de Medellín.

En primer lugar, el modelo de las cinco fuerzas de Porter (2008b) permite el empleo de un marco de referencia común para medir la presión competitiva de las industrias, teniendo en cuenta que cada una de ellas es diferente. Este modelo puede explicarse mediante cinco factores: la rivalidad entre competidores existentes, la amenaza de nuevos competidores entrantes, el poder de negociación de los proveedores, el poder de negociación de los compradores y la amenaza de productos o servicios sustitutos (ver figura 1).

Figura 1. Modelo de las cinco fuerzas de Porter

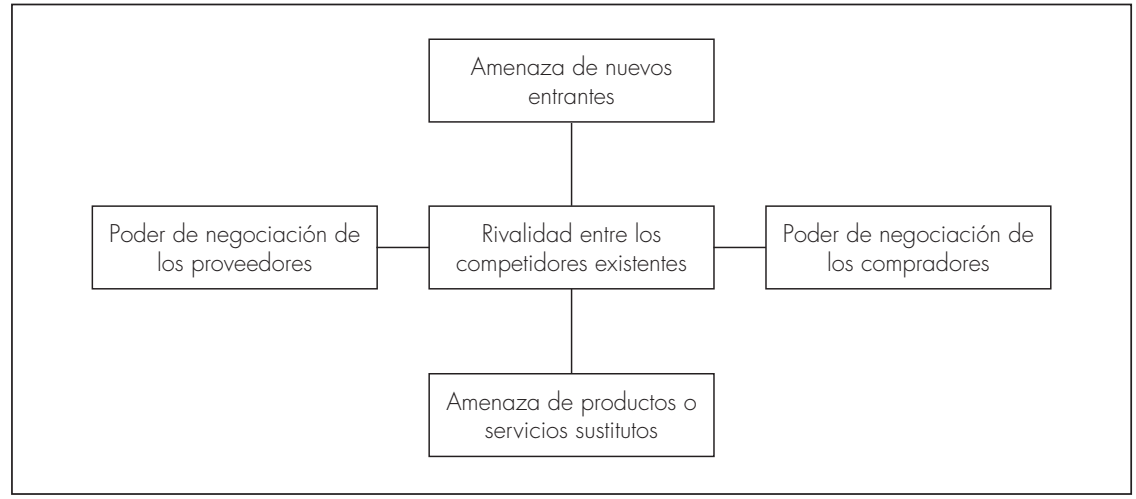

Fuente: modificado del gráfico de Porter (2008b, p. 80). 
En segundo lugar, se utilizó el diamante de la competitividad de Porter (1990) para la comparación del posible clúster en Bucaramanga con el que está ubicado en la ciudad de Medellín. Este identifica cuatro atributos genéricos que restringen o aportan a la creación de ventajas competitivas. Estos son: condiciones de losfactores; condicionesdelademanda; industrias relacionadas y de apoyo, y estrategia, estructura y rivalidad de la firma (ver figura 2).

Figura 2. Modelo del diamante de la competitividad de Porter

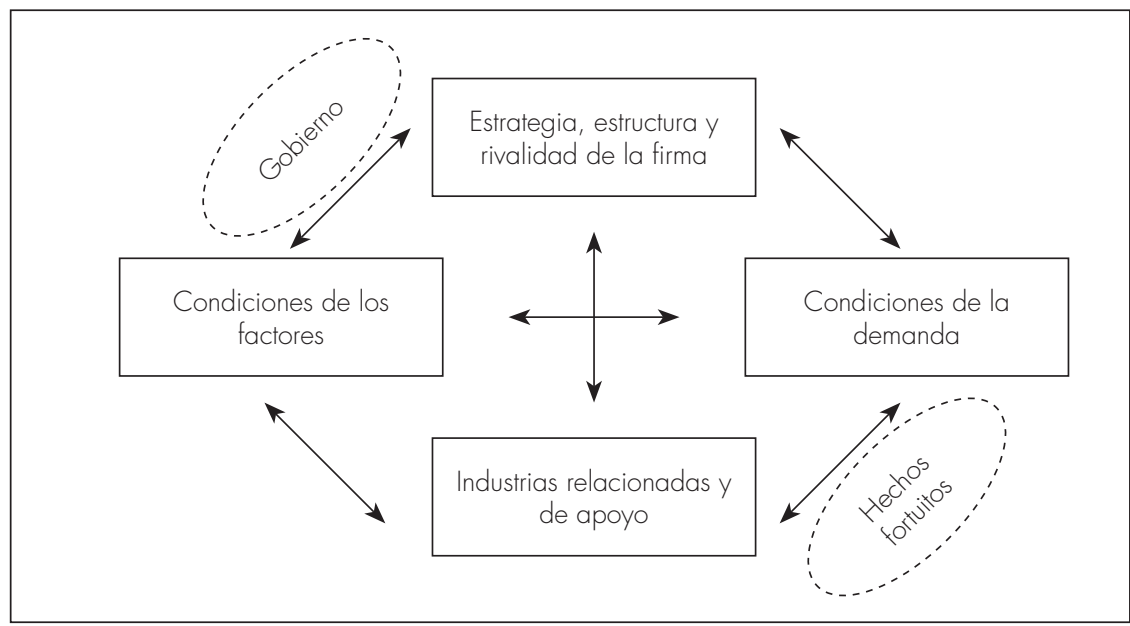

Fuente: modificado del gráfico de Porter (1990, p. 78).

Estas metodologías, en general, permiten diagnosticar y evaluar las diferentes variables externas e internas que definen la competitividad del sector de confecciones en Bucaramanga y su área metropolitana. Las primeras tienen que ver con aspectos no controlables de cada empresa, es decir, con el conjunto de tendencias e intervenciones de entes ajenos a las mismas. Estas se encuentran definidas por la balanza comercial, la generación de empleo, el grado de interdependencia entre las empresas y el grado de apoyo por parte del Gobierno. Las segundas se refieren a aspectos inherentes a la actividad empresarial. Tienen que ver con la cultura organizacional, la estructura y la composición empresarial, el grado de especialización de la mano de obra y el nivel de desarrollo tecnológico. Unas y otras, en conjunto, sirven como parámetro para calificar y comparar el sector de confecciones en Santander con el principal punto 
de referencia de este, es decir, el ubicado en el Departamento de Antioquia.

La recolección de datos para esta investigación se hizo a partir de fuentes primarias, en particular, a través de encuestas aplicadas a empresarios y entrevistas realizadas a expertos en el sector. Se incluyeron también algunas fuentes secundarias, específicamente, informes oficiales de Inexmoda, datos y estadísticas del DANE y estudios del Instituto Municipal de Empleo y Fomento Empresarial de Bucaramanga (IMEBU) y de la Cámara de Comercio de Bucaramanga. La información obtenida fue de tipo cuantitativo y cualitativo. Esta se resumió en dos matrices: la primera en función del diamante de la competitividad de Porter y, la segunda, a partir del modelo de las cinco fuerzas competitivas, promulgado por este mismo autor.

Se seleccionaron dieciséis empresas del sector de confecciones en Bucaramanga, en donde se aplicó el cuestionario. Se hicieron también cuatro entrevistas, dos de ellas a expertos de la industria y otras dos a especialistas del sector manufacturero en general pertenecientes al DANE. La muestra se escogió por el método de conveniencia, basándose, en cuatro criterios: 1) el tamaño de las empre- sas, 2) su experiencia, 3) la posibilidad de acceso real y efectivo a las mismas y 4) la disponibilidad de la información. Investigar empresas que en su mayoría son mipymes implica asumir que, por lo general, la información disponible es reducida, de difícil acceso y, en no pocas ocasiones, inexistente. Por esta razón, se eligieron las organizaciones en las cuales era posible, efectivamente, obtener datos completos y confiables.

\section{RESULTADOS Y DISCUSIÓN}

Los resultados obtenidos sobre la caracterización del sector, están sintetizados según el modelo de las cinco fuerzas competitivas de Michael Porter. Este provee una idea acerca del atractivo de una industria en términos de su rentabilidad $y$ de la convergencia de varias fuerzas relevantes en la dinámica de la competencia. Adicionalmente, como ya se ha indicado, se utilizó el método del diamante de la competitividad de Porter para evaluar la competitividad del conglomerado en Bucaramanga y, al mismo tiempo, compararlo con su principal punto de referencia: el clúster textil-confecciones-diseño de moda en Medellín. Ambas metodologías se basaron en la información proporcionada por las encuestas y las entrevistas aplicadas. 


\section{A. Fuerzas competitivas de la industria}

Amenaza de nuevos competidores entrantes

Se evidencian bajas barreras de entrada a la industria debido, en primer lugar, a la limitada presencia de economías de escala. Esto dado que la tecnología usada por las empresas del sector no es moderna. De hecho, el $56 \%$ de estas trabaja con maquinaria que tiene más de cinco años de antigüedad. Tampoco se invierte en maquinaria que permita alcanzar volúmenes de producción mayores para satisfacer la importante demanda a nivel internacional. Esta es causa frecuente de que las compañías deban recurrir a talleres satélite o maquiladoras para cumplir con el nivel de producción. Además, los procesos productivos son tradicionales y no automatizados. No hay planeación de procesos, un aspecto preocupante si se tiene en cuenta que la inversión en tecnología, que es la base de las economías de escala y que permite que las firmas tengan unos costos más competitivos, es muy baja.

Por otra parte, la lealtad que tienen los clientes hacia la oferta de valor proporcionada por las empresas, que en gran medida tiene que ver con el posicionamiento de las mar- cas y la diferenciación de los productos, muestra que la demanda de prendas de vestir es sensible a factores como el precio, las tendencias y la innovación constante. Se evidencia, de algún modo, la débil gestión de marca puesto que menos de la mitad de las empresas (44\%) tiene alguna registrada, aunque para poder afirmar esto, de manera concluyente, es necesario hacer un estudio de percepción específico en los consumidores.

El acceso a la tecnología y los canales de distribución es alto, lo que favorece la innovación. Según la Comisión Regional de Competitividad Santander Competitivo-- en 2011, a nivel local existen alrededor de 883 empresas atendiendo los canales de distribución (entre comercializadoras mayoristas y minoristas). En el exterior, las instituciones de apoyo y las comercializadoras internacionales contribuyen a que el acceso a estos, en el extranjero, sea aún mayor.

Rivalidad entre los competidores existentes en la industria

En la encuesta, los factores 'ingreso de firmas internacionales reconocidas', 'ingreso de productos internacionales a bajo precio' y 'guerras de precios' fueron calificados por la 
mayoría de los empresarios $(75 \%$, $81 \%$ y $44 \%$, respectivamente) como amenazas de alto impacto para sus empresas (ver figura 3). Se evidencia que hay un alto nivel de rivalidad (más que de competencia), expresada en términos de número de competidores y de guerra de precios. Esto debido a que la entrada de prendas de vestir importadas de bajo costo al mercado presiona el nivel de precios de las empresas locales y las obliga a tener márgenes más bajos de utilidad (como resul- tado de tener una estructura de costos poco eficiente).

El grado de rivalidad se acentúa con la baja diferenciación de los productos en todos los subsectores (moda infantil, mujeres y hombres), consolidando una oferta uniforme. Estos factores, en conjunto, justifican el decrecimiento de la industria en los últimos años, a diferencia de los niveles de crecimiento visibles en otras pertenecientes también al sector manufacturero.

Figura 3. Consideración del nivel de amenaza de factores de la industria

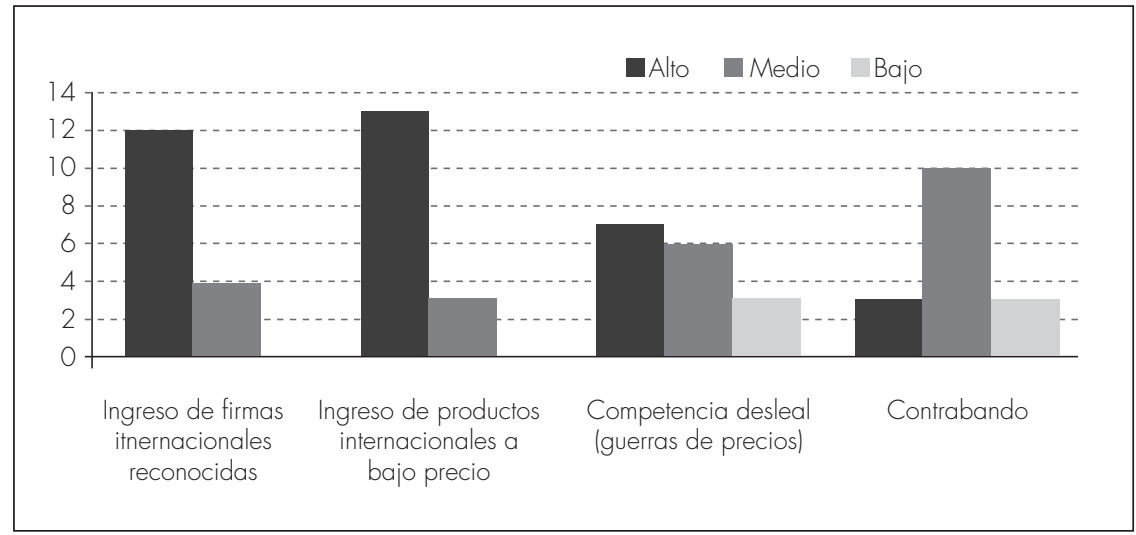

Fuente: elaboración propia.

Poder de negociación de los proveedores

A partir del trabajo realizado, se identifica que el poder de negociación de los proveedores es elevado. Los proveedores locales son pocos (64) en comparación con la canti- dad de empresas de confecciones (1179), lo que les otorga control sobre los precios. Así mismo, los proveedores internacionales, por ser más grandes en comparación con las mipymes de confecciones de $\mathrm{Bu}-$ caramanga, tienen más peso en las negociaciones. 
Poder de negociación de los compradores

El poder de negociación de los consumidores es alto, aún si se tiene en cuenta la diversidad de clientes existentes. Por un lado, están las empresas que tienen un contacto directo con el consumidor final (el $38 \%$ de ellas). Estas tienen un poder de negociación bajo con respecto a sus clientes por la facilidad de estos para encontrar productos que satisfagan su necesidad de vestuario y la gran variedad de propuestas de valor existentes en el mercado. Esto hace que el costo del cliente, por el hecho de cambiar de marca sea bajo, entre otras razones, porque el comportamiento de compra de los consumidores de este tipo de bienes está influido, en gran medida, por el cambio en los precios y el ritmo acelerado con el que se modifican las tendencias en la moda (lo que im- plica la necesidad de contar con una suficiente capacidad de innovación). Así mismo, las empresas que producen bajo la modalidad full-package (31\%), cuyos clientes son los grandes mayoristas, tienen un bajo poder de negociación frente a estos.

Finalmente, los clientes internacionales exigen no solo ciertos estándares y condiciones para la exportación, sino también un nivel de precios más bajo que las empresas de confecciones locales deben garantizar, cediéndoles, de este modo, poder de negociación. A pesar de que la estrategia que sobresale entre las empresas del sector es la diferenciación por calidad (ver figura 4), la concepción respecto a esta característica no reconoce la normalización internacional ni los requisitos para la exportación. Estas son, de hecho, las barreras no arancelarias más comunes.

Figura 4. Estrategias usadas por las empresas

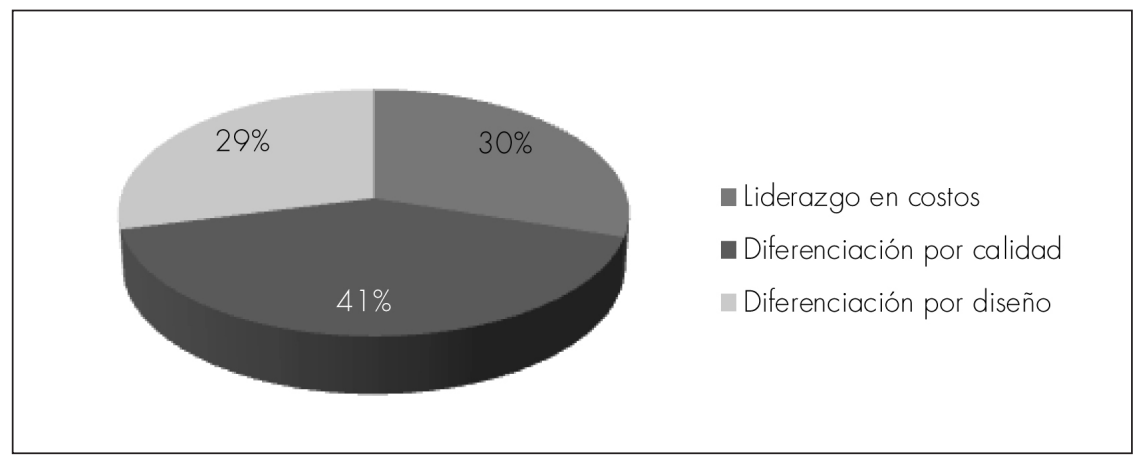

Fuente: elaboración propia. 


\section{Amenaza de productos o servicios sustitutos}

Teniendo en cuenta que las prendas de vestir son productos de uso diario y esencial, muy específicos para las personas, no existen actualmente bienes sustitutos que puedan reemplazarlos o representar una amenaza efectiva para el consumo de dichos bienes.

\section{B. Diamante de Porter}

Para evaluar la posición competitiva del sector de confecciones en Bucaramanga y, al mismo tiempo compararla con la del sector en Medellín, se resumieron las características encontradas a través de un cuadro comparativo que se observa, a continuación, en la tabla 1.

Tabla 1. Cuadro comparativo del sector de confecciones en Bucaramanga y en Medellín, según el modelo del diamante de la competitividad de Porter

\begin{tabular}{|c|c|c|c|}
\hline & & Área metropolitana de Bucaramanga & Área metropolitana de Medellín \\
\hline \multirow{14}{*}{$\begin{array}{l}\text { Condiciones } \\
\text { de los factores }\end{array}$} & \multirow{5}{*}{$\begin{array}{l}\text { Recursos } \\
\text { humanos }\end{array}$} & \multicolumn{2}{|l|}{ (-) Alta rotación de personal. } \\
\hline & & $\begin{array}{l}\text { (-) Bajo grado de especialización y cali- } \\
\text { ficación del talento humano intermedio } \\
\text { (diseñadores y patronistas). }\end{array}$ & $\begin{array}{l}\text { (+) Alto grado de especialización de } \\
\text { talento humano intermedio (diseñado- } \\
\text { res y patronistas). }\end{array}$ \\
\hline & & \multicolumn{2}{|c|}{ (-) Bajo grado de especialización y calificación del talento humano operativo. } \\
\hline & & \multicolumn{2}{|c|}{$(+)$ Amplia oferta de capacitación del talento humano. } \\
\hline & & $\begin{array}{l}\text { (-) Costos de mano de obra no competi- } \\
\text { tivos internacionalmente. }\end{array}$ & $\begin{array}{l}(+) \text { Costos de mano de obra competiti- } \\
\text { vos internacionalmente. }\end{array}$ \\
\hline & \multirow{2}{*}{$\begin{array}{l}\text { Recursos del } \\
\text { conocimiento }\end{array}$} & (-) Baja acumulación de conocimiento. & $(+)$ Alta acumulación de conocimiento. \\
\hline & & (-) Bajos niveles de Inversión en I+D. & (+) Altos niveles de inversión en I+D. \\
\hline & $\begin{array}{l}\text { Recursos de } \\
\text { capital }\end{array}$ & $\begin{array}{l}\text { (-) Bajo acceso a crédito y otras fuentes } \\
\text { de financiación a mipymes. }\end{array}$ & $\begin{array}{l}(+) \text { Alto acceso a crédito y otras fuentes } \\
\text { de financiación a mipymes. }\end{array}$ \\
\hline & $\begin{array}{l}\text { Recursos } \\
\text { Físicos }\end{array}$ & \multicolumn{2}{|l|}{ (+) Proximidad a los mercados extranjeros. } \\
\hline & \multirow{5}{*}{$\begin{array}{l}\text { Recursos de } \\
\text { infraestructura }\end{array}$} & $\begin{array}{l}\text { (-) Bajo nivel de cantidad y calidad de } \\
\text { la infraestructura vial. }\end{array}$ & $\begin{array}{l}(+) \text { Alto nivel de cantidad y calidad de } \\
\text { la infraestructura vial. }\end{array}$ \\
\hline & & \multicolumn{2}{|l|}{ (-) Bajo nivel de infraestructura aérea. } \\
\hline & & \multicolumn{2}{|c|}{ (+) Gran disponibilidad de infraestructura de TIC. } \\
\hline & & \multicolumn{2}{|c|}{$\begin{array}{l}(+) \text { Gran cantidad de proyectos de mejoramiento de la calidad de la infraestruc- } \\
\text { tura vial. }\end{array}$} \\
\hline & & \multicolumn{2}{|l|}{ (+) Calidad de los servicios públicos. } \\
\hline
\end{tabular}




\begin{tabular}{|c|c|c|c|}
\hline & & Área metropolitana de Bucaramanga & Área metropolitana de Medellín \\
\hline \multirow{3}{*}{$\begin{array}{l}\text { Condiciones } \\
\text { de los factores }\end{array}$} & \multirow{3}{*}{$\begin{array}{l}\text { Recursos } \\
\text { tecnológicos }\end{array}$} & $\begin{array}{l}\text { (-) Bajo nivel de planeación y sistema- } \\
\text { tización de los procesos productivos. }\end{array}$ & $\begin{array}{l}(+) \text { Las empresas están comenzando } \\
\text { a planear y sistematizar los procesos } \\
\text { productivos. }\end{array}$ \\
\hline & & \multicolumn{2}{|c|}{ (-) Baja actualización de software, maquinaria y equipo. } \\
\hline & & $\begin{array}{l}\text { (-) Altos niveles de desperdicio de } \\
\text { recursos. }\end{array}$ & $\begin{array}{l}\text { (+) Aprovechamiento de residuos en } \\
\text { la fuente. }\end{array}$ \\
\hline \multirow{6}{*}{$\begin{array}{l}\text { Condiciones } \\
\text { de la demanda }\end{array}$} & \multirow{2}{*}{$\begin{array}{l}\text { Segmentación } \\
\text { de la demanda }\end{array}$} & \multicolumn{2}{|l|}{ (+) Gran tamaño de mercado. } \\
\hline & & \multicolumn{2}{|c|}{ (+) Altos niveles de segmentación de la demanda nacional y extranjera. } \\
\hline & & \multicolumn{2}{|c|}{$(+)$ Alta sofisticación y poder adquisitivo de la demanda extranjera. } \\
\hline & Sofisticación & \multicolumn{2}{|c|}{ (-) Baja sofisticación de la demanda interna y sensibilidad al precio. } \\
\hline & $\begin{array}{l}\text { de compra- } \\
\text { dores }\end{array}$ & \multicolumn{2}{|c|}{$\begin{array}{l}\text { (+) Creciente interés de los consumidores por la moda y las tendencias } \\
\text { (incrementando la frecuencia de compra). }\end{array}$} \\
\hline & & \multicolumn{2}{|c|}{ (-) Presión sobre los precios, ejercida por las importaciones económicas. } \\
\hline \multirow{8}{*}{$\begin{array}{l}\text { Estrategia, } \\
\text { estructura y } \\
\text { rivalidad de la } \\
\text { firma }\end{array}$} & \multirow{3}{*}{ Estrategia } & \multicolumn{2}{|c|}{$(+)$ Creciente interés por el desarrollo de marcas propias. } \\
\hline & & $\begin{array}{l}\text { (-) Bajo nivel actual de diferenciación } \\
\text { de los productos. }\end{array}$ & $\begin{array}{l}(+) \text { Énfasis en la diferenciación de los } \\
\text { productos. }\end{array}$ \\
\hline & & $\begin{array}{l}\text { (-) Cultura empresarial de renuencia al } \\
\text { cambio y aversión al riesgo. }\end{array}$ & $(+)$ Cultura empresarial emprendedora. \\
\hline & & \multicolumn{2}{|l|}{ (-) Predominancia de microempresas. } \\
\hline & Estructura & \multicolumn{2}{|c|}{ (+) Disminución de la presencia de talleres satélite o maquilas. } \\
\hline & & \multicolumn{2}{|c|}{ (-) Presencia de informalidad laboral y contrabando. } \\
\hline & & \multicolumn{2}{|c|}{$(+)$ Alto nivel de competencia por calidad y precio. } \\
\hline & Rivalidad & $\begin{array}{l}\text { (-) Bajo grado de asociación y } \\
\text { cooperación. }\end{array}$ & $\begin{array}{l}(+) \text { Alto grado de asociación y } \\
\text { cooperación. }\end{array}$ \\
\hline \multirow{6}{*}{$\begin{array}{l}\text { Industrias } \\
\text { relacionadas y } \\
\text { de apoyo }\end{array}$} & \multirow{4}{*}{$\begin{array}{l}\text { Proveedores } \\
\text { (insumos, } \\
\text { maquinaria y } \\
\text { servicios) }\end{array}$} & $\begin{array}{l}\text { (-) Baja producción de insumos a nivel } \\
\text { local. }\end{array}$ & $\begin{array}{l}(+) \text { Producción de insumos a nivel } \\
\text { local. }\end{array}$ \\
\hline & & \multicolumn{2}{|c|}{ (+) Alta disponibilidad de insumos y materias primas a nivel local y regional. } \\
\hline & & \multicolumn{2}{|l|}{ (-) Baja oferta de maquinaria del sector. } \\
\hline & & $\begin{array}{l}\text { (-) Baja disponibilidad de empresas que } \\
\text { ofrecen servicios de calidad (teñido, } \\
\text { bordado, estampado, procesos textiles, } \\
\text { etc.). }\end{array}$ & $\begin{array}{l}\text { (+) Alta disponibilidad de empresas que } \\
\text { ofrecen servicios de calidad (teñido, } \\
\text { bordado, estampado, procesos textiles, } \\
\text { etc.). }\end{array}$ \\
\hline & & \multicolumn{2}{|c|}{ (+) Disponibilidad de programas académicos de apoyo al sector. } \\
\hline & Educativas & \multicolumn{2}{|c|}{$\begin{array}{l}\text { (+) Interés del sector educativo por promover la generación de conocimiento en } \\
\text { el sector. }\end{array}$} \\
\hline
\end{tabular}




\begin{tabular}{|c|c|c|c|}
\hline & & Área metropolitana de Bucaramanga & Área metropolitana de Medellín \\
\hline \multirow{3}{*}{$\begin{array}{l}\text { Industrias } \\
\text { relacionadas } \\
\text { y de apoyo }\end{array}$} & \multirow{3}{*}{$\begin{array}{l}\text { Entidades } \\
\text { de fomento } \\
\text { empresarial }\end{array}$} & $\begin{array}{l}\text { (+) Gran acompañamiento y coope- } \\
\text { ración de entidades como la Cámara } \\
\text { de Comercio, ProExport, IMEBU e } \\
\text { Inexmoda. }\end{array}$ & $\begin{array}{l}(+) \text { Gran acompañamiento y coope- } \\
\text { ración de entidades como la Cámara } \\
\text { de Comercio, ProExport, Inexmoda, } \\
\text { Ascoltex, Asotextil, etc. }\end{array}$ \\
\hline & & $\begin{array}{l}\text { (+) Presencia de entidades de agremia- } \\
\text { ción como Acopi, Buconsa, CDP de la } \\
\text { Confección, etc. }\end{array}$ & $\begin{array}{l}(+) \text { Presencia de entidades de } \\
\text { agremiación como Cedemoda, Acopi } \\
\text { Antioquia, etc. }\end{array}$ \\
\hline & & $(+)$ Ferias EIMI y SFW. & $\begin{array}{l}(+) \text { Ferias Colombiamoda, Colombia- } \\
\text { tex, Feriamoda. }\end{array}$ \\
\hline
\end{tabular}

Fuente: elaboración propia.

De acuerdo con la tabla 1, la posición competitiva del sector en Medellín es mejor que la del sector en Bucaramanga. Esto al tener más aspectos positivos (señalados con el signo "+") que son afectados por la presencia de una aglomeración o clúster. En efecto, el sector de confecciones en Bucaramanga evidencia un comportamiento desfavorable en las variables que tienen que ver con la capacitación y especialización del talento humano, el costo de la mano de obra, los niveles de acumulación del conocimiento, la inversión en investigación y desarrollo, la planeación de procesos, la diferenciación de los productos, el grado de asociación y cooperación de las empresas del sector y la cultura organizacional en general.

A pesar de que el clúster es un concepto amplio, flexible y que carece de un modelo universal y absoluto que garantice el crecimiento económico en donde quiera que se aplique (Martin \& Sunley, 2003), este se presenta, efectivamente, como una posible solución a la situación actual del sector de confecciones en Santander.

En primer lugar, la situación actual del sector de confecciones y producción textil muestra un notable decaimiento en los últimos años, de acuerdo con la visible disminución de los flujos de producción y de exportaciones. Este comportamiento del sector se debe principalmente a tres aspectos: 1) rezago tecnológico, 2) baja inversión en investigación y desarrollo y 3) bajo nivel de emprendimiento. Además, se evidencian algunos factores en el ambiente externo que convierten a dichas carencias en puntos neurálgicos a la hora de lograr una competitividad general del sector. Estos tienen que ver con la apertura económica que ha experimentado Colombia y el mundo en los últimos años. Este fenómeno ha ocasionado un aumento importante de las importaciones tanto de prendas de vestir de bajo 
precio, como de algunas pertenecientes a reconocidas marcas en el mercado internacional.

Además, están latentes aspectos de la cultura empresarial del departamento como la renuencia al cambio, el bajo interés de exploración de nuevos mercados y una rivalidad entre los participantes de la industria que no fomenta la competitividad sino que, por el contrario, genera un ambiente en el que los empresarios prefieren rendimientos mínimos de sus empresas. Esto sin importar que al asociarse sea posible lograr un mayor beneficio para las empresas de la industria como un todo al conseguir, por ejemplo, costos más bajos de materia prima, facilidades de acceso a maquinaria o el cumplimiento de las cuotas mínimas de exportación.

En segundo lugar, como se mencionó anteriormente, existen varios casos de éxito de generación de clústeres para mejorar la situación del sector de confecciones y producción textil en países desarrollados, como Canadá, y en vías de desarro1lo, como China y México. En todos los casos, los niveles de producción, empleo y exportaciones mejoraron. Esto además de que se enfatizaba en la inversión en tecnología e innovación, de la mano de una adecuada integración de la cadena productiva.
Al tener varias perspectivas globales y un caso de éxito nacional, como el de Medellín, se evidencian los beneficios de dicho modelo de integración que, si bien parte de un 'ideal', puede llegar a ser aplicable a diferentes realidades económicas, dada la propia amplitud del concepto.

Para cuantificar el impacto y la factibilidad del clúster en la situación económica de la región, y teniendo en cuenta como primera medida la balanza comercial, se evidencia que si bien la crisis del sector a nivel nacional ha impactado negativamente al mismo, Medellín tuvo una balanza comercial superavitaria de alrededor de USD 171 millones en 2010. Esta contrasta con la de Bucaramanga (Santander), un área geográfica que no cuenta con una integración formal (clúster) de la cadena productiva, puesto que la balanza comercial del sector en 2010 fue deficitaria en USD 580000 .

Por otra parte, en los primeros cuatro años de la creación del clúster (20012005) las exportaciones de Antioquia se incrementaron en un $11 \%$. Esto demuestra un gran beneficio para el sector, en la medida en que la integración le permitió responder a las oportunidades planteadas en materia de comercio exterior. Además, la evolución del personal ocupado en el sector de confecciones de 2009 
a 2010 varió de manera un poco menos negativa para Antioquia que para Santander. En el primer departamento, hubo un 13,6\% menos de personas laborando en este sector, en el segundo, esta cifra alcanzó un $14,9 \%$.

Si se miran las cifras de producción, por otra parte, es evidente que el clúster que integra la cadena productiva textil-confecciones en Medellín ha contribuido en gran medida para que los niveles de producción y de ventas hayan aumentado a 2010. Esto diverge con el comportamiento de esos mismos niveles en el departamento de Santander. Así, la producción de prendas de vestir aumentó $7,9 \%$ en Antioquia, mientras que, en Santander, disminuyó un 9,3\%. Las ventas en Antioquia, por su parte, aumentaron un 7,4\%; en Santander, por el contrario, disminuyeron un $6,8 \%$.

En términos generales, el sector actualmente no tiene una posición competitiva favorable. No obstante, estas son condiciones que se pueden desarrollar por medio de un modelo como el clúster, ya que, por la importancia del sector en la economía local, sería un gran desperdicio de recursos desmotivar o desincentivar la industria de las confecciones en el departamento. Uno de los beneficios del clúster, por ejemplo, es que al proponer una concentración de varias industrias relacionadas en un área metropolitana, se generan no solo más ingresos, sino también tasas de ocupación más altas (Spencer, Vinodrai, Gertler, \& Wolfe, 2010). El sector de confecciones y diseño de modas en Medellín, por su parte, ha adquirido cierto nivel de innovación que, según Albarracín y Pérez (2012), es el factor que produce un mayor impacto positivo en las mipymes y genera una mayor flexibilidad y adaptabilidad al cambio (Drucker, 1986).

Un aspecto en el que se deben realizar mayores esfuerzos es el acompañamiento, por parte de entidades como las instituciones educativas, las organizaciones competentes de apoyo empresarial y las entidades bancarias. Hay que hacer hincapié, preferiblemente, en las primeras. Estas instituciones, al ser fuentes en materia de capacitación, pueden contribuir efectivamente a romper las barreras que impiden el aprendizaje en las mipymes y facilitar además la creación de redes de colaboración a su alrededor (Madrigal Torres, Vargas, \& Torres, 2012). Por lo demás, industrias relacionadas, como el calzado y la joyería, que también conservan una presencia importante en la región y que tienen en común los canales de comunicación y distribución, podrían ser 
incluidos también en el clúster, con el objetivo de producir sinergias, tal como lo sugiere Porter (1998).

Teniendo en cuenta que el aprendizaje y la generación de conocimiento en las organizaciones son procesos fundamentales para el logro de la innovación en las mismas, y que cualquier iniciativa de los empleados, gerentes o directores del clúster puede ser benéfica para crear estrategias cuando hay un ambiente de participación (Valélia \& De Andrade Jú, 2012), conviene exaltar la importancia de la capacitación y la especialización del talento humano en todos los niveles de la organización. Esto para que dicha producción de saber sea de alto impacto en las organizaciones dentro del contexto de las redes empresariales.

De acuerdo con lo anterior, y ya que las empresas del sector de confecciones en Colombia parecen no estar en capacidad de apostarle a una ventaja competitiva a través de una estrategia de liderazgo en costos (Carmona \& Gil Quintero, 2010), los esfuerzos de estas compañías podrían enfocarse hacia la formación del talento humano, más aún por tratarse los suyos de bienes intensivos en mano de obra. Estas acciones podrían traducirse no solo en una mayor productividad, sino tam- bién en un mejor desempeño innovador (Hauknes, 1999; Rodríguez \& Álvarez Giraldo, 2011; Vinding, 2006). Ellas podrían aportar así a la obtención de un mayor valor agregado para dichos productos. Esto a partir de la identificación de que la ventaja competitiva de estas firmas puede estar enfocada, más bien, en una estrategia de diferenciación.

Si se fija la creación de valor agregado como el objetivo principal, salta a la vista la necesidad de mejorar los modelos de negocio, eliminando la maquila como una alternativa que, si bien produce mayor volumen de producción y menores costos (Cota, 2009), en el mediano y largo plazo genera una destrucción de valor (Carmona \& Gil Quintero, 2010). De este modo, el sector de confecciones en Colombia podría apuntarle principalmente a una recuperación de la balanza comercial por medio de la exportación de productos altamente diferenciados y de calidad. Una acción que, tal vez, en términos de volúmenes, represente menos, pero que en términos de dinero puede ser significativo.

Finalmente, se resalta el papel que juega el Gobierno local y sus instituciones de apoyo al promover políticas, planes y agendas de implementación de clústeres, tal como proponen Hernández y Montalvo 
(2012). Esto al señalar que, en tiempos de pérdida de competitividad a nivel mundial, las políticas públicas en materia de negocios deben ser más agresivas, persistentes y consistentes, identificando las actividades económicas que se pueden integrar en una cadena productiva para mejorar la competitividad de una región.

\section{CONCLUSIONES}

En el estudio presentado se evidencia la factibilidad de la creación y consolidación del clúster de confecciones en el área metropolitana de Bucaramanga, puesto que esto puede contribuir de manera importante al mejoramiento de la competitividad del sector en la región. Como resultado del trabajo de investigación realizado, se encontraron factores que sugieren que la masa empresarial y las condiciones de la industria y del mercado tienen potencial para desarrollar el clúster. Se destacan entre ellos: 1) la presencia de empresas en todos los eslabones de la cadena productiva y de apoyo, 2) la proximidad a mercados internacionales y 3) el gran tamaño de mercado (local, nacional e internacional). Estos elementos permiten, al mismo tiempo, desarrollar factores de éxito básicos como el nivel de inversión en maquinaria y la actualización tecnológica, la capacitación y la especialización del talento humano y la generación de una necesaria cultura organizacional que esté más abierta a la creación, al cambio $\mathrm{y}$ al riesgo.

\section{REFERENCIAS}

Albarracín, E. J. G., \& Pérez, D. G. (2012). Impacto de la innovación sobre el rendimiento de la MIPYME: un estudio empírico en Colombia. Estudios Gerenciales, 28(122), 11-28.

Barnes, L., \& Lea-Greenwood, G. (2006). Fast fashioning the supply chain: Shaping the research agenda. Journal of Fashion Marketing and Management, 10(3), 259-271.

Barsoumian, S., Riggio, R., Severin, A., \& Van der Spek, T. (2011). Eco-innovation in cluster organisations in the chemical and textile-clothing-leather sectors: A qualitative review. Bruselas: Greenovatd, Europe EEIG.

Campaniaris, C., Hayes, S., Jeffrey, M., \& Murray, R. (2011). The applicability of cluster theory to Canada's small and mediumsized apparel companies. Journal of Fashion Marketing and Management, 15(1), 8-26.

Carmona, R. J., \& Gil Quintero, J. D. (2010). Competitividad y retos en la productividad del clúster textil confección. Diseño y moda 
en Antioquia. Revista Ciencias Estratégicas, 16(20), 247-263.

Cota, Y., M. d. R. (2009). Estructura organizacional de las empresas de la confección de Zapotlanejo, Jalisco. Problemas del Desarrollo, 35(138), 155-176.

Compite 360. (s.f.). Composición de la masa empresarial. Recuperado de http://www.compite360.com/.

DANE (2010). Informe de coyuntura económica regional del Departamento de Santander [en línea]. Recuperado de http://www.dane. gov.co/files/icer/2010/santander_icer_10.pdf

Drucker, P. F. (1986). Innovation and entrepreneurship: Practices and principles. The Journal of Continuing Higher Education, 34(1), 22-23.

Hauknes, J. (1999). Norwegian input-output clusters and innovation patterns. En OECD (Ed.), Boosting innovation the cluster approach. París: OECD.

Hernández,C.,\&Montalvo,C.(2012). Entrepreneurial clusters in China and Mexico-implications for competitiveness. Revista Globalización, Competitividad y Gobernabilidad, 6(1), 55-90.

Inexmoda (2012). Presentación en el pabellón del conocimiento de Colombia Moda 2012. Outlook del sector. Recuperado de http:// observatorioeconomico.inexmoda.org.co/Portals/0/Presentaci $\%$
C 3 \% B 3 n $\% 20$ Pabe $11 \%$ C $3 \%$ B3n $\% 20$ del $\% 20$ conocimiento $\%$ 20Colmoda\%202012.web.pdf

Lee, J., \& Karpova, E. E. (2011). The US and Japanese apparel demand conditions: Implications for industry competitiveness. Journal of Fashion Marketing and Management, 15(1), 76-90.

Madrigal Torres, B. E., Vargas, R. A., \& Torres, R. M. (2012). El emprendedor y su capital social: caso el clúster del software en jalisco. Revista Internacional Administración \& Finanzas, 5(4), 107-120.

Martin, R., \& Sunley, P. (2003). Deconstructing clusters: Chaotic conceptorpolicy panacea? Journal of economic geography, 3(1), 5-35.

Mercado, C. H. J., Fontalvo, H. T. J., \&DelaHozGranadillo, E.(2011). Análisis comparativo entre las cadenas productivas del sector textil-confecciones de la provincia de Jiangsu-China y el departamento del Atlántico-Colombia. Ingeniare. Revista Chilena de Ingeniería, 19, 429-441.

Navarro, A. M. (2003). Análisis y políticas de clusters: teoría y realidad. Ekonomiaz: Revista Vasca de Economía, 53(2), 14-49.

Porter, M. E. (1990). The competitive advantage of nations. Harvard Business Review, 68(2), 73-91.

Porter, M. E. (1998). Clusters and the new economics of competi- 
tion. Harvard Business Review, 76(6), 77-90.

Porter, M. E. (2008a). On competition (updated and expanded ed.). Boston: Harvard Business School Pub.

Porter, M. E. (2008b). The five competitive forces that shape strategy. Harvard Business Review, 86(1), 78-93.

Rodríguez, F. B., \& Álvarez Giraldo, C. M. (2011). El talento humano y la innovación empresarial en el contexto de las redes empresariales: el clúster de prendas de vestir en Caldas-Colombia. Estudios Gerenciales, 27(119), 209-232.

Silva, F. F., Feitosa, M. G. G., \& Aguiar, V. d. S. M. (2012). Uma reflexão sobre as relações de parceria nos APLs de Confecções do Agreste Pernambucano como elemento disseminador da inovação em redes interorganizacionais. RAM. Revista de Administração Mackenzie, 13, 206-235. Spencer, G. M., Vinodrai, T., Gertler, M. S., \& Wolfe, D. A. (2010). Do clusters make a difference? Defining and assessing their economic performance. Regional Studies, 44(6), 697-715.

Valélia, S. A., \& De Andrade Jú, P. P. (2012). Analysis of aspects of innovation in a Brazilian cluster. Journal of Technology Management\&Innovation, 7(3), 207-212. Vinding,A. L. (2006). Absorptive capacity and innovative performance: A human capital approach. Economics of Innovation \& New Technology, 15(4/5), 507-517. 